Kansas State University Libraries

New Prairie Press

\title{
MODELING AND DESIGN TO DETECT INTERACTION OF INSECTICIDES, HERBICIDES AND OTHER SIMILAR COMPOUNDS
}

Timothy E. O'Brien

Follow this and additional works at: https://newprairiepress.org/agstatconference

Part of the Agriculture Commons, and the Applied Statistics Commons

\section{c) (1) () $\Theta$}

This work is licensed under a Creative Commons Attribution-Noncommercial-No Derivative Works 4.0 License.

\section{Recommended Citation}

O'Brien, Timothy E. (2003). "MODELING AND DESIGN TO DETECT INTERACTION OF INSECTICIDES, HERBICIDES AND OTHER SIMILAR COMPOUNDS," Conference on Applied Statistics in Agriculture. https://doi.org/10.4148/2475-7772.1194

This is brought to you for free and open access by the Conferences at New Prairie Press. It has been accepted for inclusion in Conference on Applied Statistics in Agriculture by an authorized administrator of New Prairie Press. For more information, please contact cads@k-state.edu. 


\title{
MODELING AND DESIGN TO DETECT INTERACTION OF INSECTICIDES, HERBICIDES AND OTHER SIMILAR COMPOUNDS
}

\author{
Timothy E. O'Brien \\ Loyola University Chicago, Department of Mathematics and Statistics \\ 6525 N. Sheridan Road, Chicago, IL 60626 USA \\ tobrien@math.luc.edu
}

\begin{abstract}
This paper discusses model and experimental design aspects of agricultural studies aimed at discerning antagonism or synergy between two or more insecticides, herbicides, or other similar compounds. The developed methods involve a broad class of generalised nonlinear models, which are easily fitted to data using popular statistical packages such as the NLMIXED procedure in $\mathrm{SAS}^{\circledR}$ software. Sample computer code is given in the Appendix.
\end{abstract}

Keywords - antagonism, combination index, experimental design, generalized nonlinear models, isobolograms, optimal design, phenolic acids, synergy.

\section{Introduction}

Nonlinear models, such as dose-response models, are often used in agricultural settings to relate the response of interest to the concentration of an herbicide, insecticide or other similar compounds such as a phenolic acids. In settings where combinations of such similar compounds are applied to the given experimental units, interest often centers on the detection of any relevant interaction such as an additive effect (synergy), sub-additive effect (antagonism) or independent effect. Statistical models useful for the detection of such interactions are discussed in Finney $(1952,1971,1978)$ and Greco et al (1995), experimental design strategies for interaction are given in Abdelbasit and Plackett (1982), Greco and Tung (1991) and O'Brien (2003a, 2003b), and Bates and Watts (1988), Seber and Wild (1989) and McCullagh and Nelder (1989) cover generalized nonlinear modelling. Additional references include Chou and Talalay (1981), Berenbaum (1989), Barton et al (1993), Khinkis and Greco (1993), Greco et al (1995), Dawson et al (2000), Hutchinson et al (2000) and Govindarajulu (2001). Our focus here is to provide new modelling and experimental design methods useful for the detection of synergy/antagonism with applications to agricultural settings.

\section{Preliminaries}

Before the experimental design can be chosen and before the assessment of interaction between two similar compounds can be made, several important concerns need to be addressed - an appropriate model must be chosen, the choice of the proper (dose or concentration) scale must be made and the relative potency of the two compounds must be estimated. 


\subsection{The Choice of the Statistical Model}

We consider here parametric statistical models from the general class of generalized linear or nonlinear models, meaning that assumptions must be made as to the underlying distribution of the response variable(s), the mean function and the link function. Indeed, this restriction is easily relaxed to include all nonlinear models for which one can specify a likelihood; thus, distributions are allowable outside the exponential family such as the Weibull distribution. One model useful for modelling synergy is the so-called Finney5 model (Finney, 1971, Chap. 11). For this model, similar compounds (e.g., herbicides, pollutants, etc.) $\mathrm{A}$ and $\mathrm{B}$ in amounts $\mathrm{x}_{1}$ and $\mathrm{x}_{2}$ can be related to a response $\mathrm{Y}$ by first calculating the effective dose,

$$
z=x_{1}+\theta_{4} x_{2}+\theta_{5}\left(\theta_{4} x_{1} x_{2}\right)^{1 / 2}
$$

where $\theta_{4}$ is the relative potency parameter and $\theta_{5}$ is the coefficient of synergy, and then by using a parametric model such as the 3-parameter log-logistic model (LL3)

$$
\eta=E(Y)=\theta_{1} /\left\{1+\left[\left(x_{1}+\theta_{4} x_{2}+\theta_{5}\left(\theta_{4} x_{1} x_{2}\right)^{1 / 2}\right) / \theta_{2}\right]^{\theta 3}\right\}
$$

Note that $\theta_{1}$ is the expected response at zero dose level, that $\theta_{2}$ and $\theta_{3}$ are the $\mathrm{LD}_{50}$ and slope parameters respectively, and should a non-zero lower asymptote $\left(\theta_{7}\right)$ be required, then we could use the 4-parameter log-logistic model (LL4) in place of the LL3 model. For these models, the associated curve is descending (as the effective dose increases) whenever $\theta_{3}$ is positive, so that this model may be useful to describe the antagonistic effect of two phenolic acids on the growth of cucumber seedlings, the synergistic effect of two pollutants in terms of crop yield, etc.

A minor adaptation of this function, called the Finney 4 model - useful for binomial doseresponse data - assumes that the response variable has a binomial distribution with a vector of n's (with sample size depending on the dose levels), and with 'success' probability

$$
\mathrm{p}=\mathrm{t} /(1+\mathrm{t})
$$

for $t$ and $z$ defined in equations (2.1) and (2.2). We point out that for the chosen parameterization (where $\theta_{2}$, the $\mathrm{LD}_{50}$, is a model parameter), these models are indeed generalized nonlinear models - models which are finding more and more recent applications (see e.g. Cox (1992)). By way of illustration, since for positive $\theta_{3}$, the curve associated with equation (2.3) is increasing with $z$, this model is useful, for example, for modelling the proportion of dead insects associated with two insecticides; applications of this sort are discussed in section 3.

Although the Finney model is useful for a modelling interaction in some cases, this model has proven to be too restrictive for several datasets. Whenever the data is chosen using so-called ray designs, an important generalization, called the separate ray model, is to fit separate nonlinear curves along each ray, each with a potentially different slope and possibly different scale (see section 2.2 below). Here, several combinations of points are chosen along each of $r$ rays, where 
the $\mathrm{x}$-axis $\left(\mathrm{x}_{2}=0\right)$ is taken as the first ray, the $\mathrm{y}$-axis $\left(\mathrm{x}_{1}=0\right)$ is taken as the second ray, and where the $\mathrm{r}^{\text {th }}$ ray corresponds to the line

$$
\mathrm{x}_{2}=\mathrm{c}_{\mathrm{r}} \mathrm{x}_{1}
$$

for the chosen (R-2) slopes $\mathrm{c}_{\mathrm{r}}$ (for $\left.\mathrm{r}=3,4, \ldots \mathrm{R}\right)$. The slopes $\mathrm{c}_{3}, \ldots, \mathrm{c}_{\mathrm{R}}$ are fixed a priori, and correspond to the so-called interior rays. Thus, for example for binomial data, equation (2.3) is fit along each ray. It is straightforward to show that when the corresponding slopes are equal and the $\mathrm{LD}_{50}$ 's follow a specific relationship, then the separate ray model collapses to the Finney model; this result is established in Appendix A.1. The separate ray model is discussed further in Section 3 in the context of effective fractions and combination indices.

We also point out the importance of testing model assumptions (by examining and testing residuals, etc.) once the model and design are chosen, the data is obtained, and the model parameters are estimated. For example, for some datasets for which continuous Gaussian responses are assumed, we have found it useful to model variances according to the assumed variance function, $\operatorname{var}(\mathrm{Y})=\sigma^{2} \eta^{\phi}$, where $\eta=\mathrm{E}(\mathrm{Y})$ and $\phi$ is an additional variance parameter to be estimated from the data. Other examples of extending the basic model assumptions are the use of the beta-binomial distribution in place of the binomial distribution and the use of the negative binomial or the generalized Poisson distribution in place of the Poisson distribution whenever overdispersion is a concern for a given dataset.

\subsection{The Choice of Scale}

Researchers and beginning students often become understandable confused when textbooks such as Myers et al (2001:114) fit a logistic regression curve using both the dose and the log-dose scales to a given dataset and give no clear logic behind how the choice of scale can and should be made. Indeed, in many cases, statistical modellers and practitioners are uncertain as to the correct scale to use. Even in cases where the doses are chosen according to a geometric pattern and where one might be tempted to use the log-concentration to fit the logistic curve, we highlight the fact that since the logistic curve only represents a model of the actual relationship, this temptation needn't be necessarily followed (nor appropriate) in all situations. One way to circumvent this quandary is to have the data "choose" the correct scale by introducing a BoxCox scale model parameter which is then estimated. Thus we replace the probability in equation (2.3) by

$$
\mathrm{p}=\mathrm{ex} /(1+\mathrm{ex})
$$

where "ex" $=\exp \left\{\theta_{3}\left[z(x)-z\left(\theta_{2}\right)\right]\right\}$ and where

$$
z(x)=\left[x^{\theta 6}-1\right] / \theta_{6}
$$

so that $\theta_{6}$, the Box-Cox parameter, is indeed an additional parameter to be estimated. As this additional parameter nears zero, the log-dose scale is indicated for the logistic (or other) model, 
and as it nears one, the dose-scale is deemed more appropriate, though our practice is to include other values as well. For the referenced Myers et al exercise, since the $95 \%$ confidence interval for the Box-Cox parameter includes zero but does not include one, we conclude that these data suggest the use of the log-dose and the model in the form of (2.3). We have also found this step an important one in the assessment of both relative potency and of drug synergy. Additional illustrations of the importance of the scale parameter include Example 2.3.1 and the examples given in sections 3 and 4 .

\subsection{Estimating the Relative Potency}

Before an experiment can be designed to test for the nature of the interaction between two similar compounds or drugs, the relative potency of the two drugs should first be estimated. Thus, if separate logistic model functions of the form of equation (2.3) are fit to each drug, then we can define the relative potency in terms of the respective $\mathrm{LD}_{50}$ 's, by

$$
\rho=\theta_{21} / \theta_{22}
$$

where $\theta_{21}$ is the $L D_{50}$ associated with drug $A$ and $\theta_{22}$ is the $L D_{50}$ associated with drug $B$. Note that if instead the $\mathrm{LD}_{75}$ 's (the respective doses at which $\mathrm{p}=75 \%$ for model (2.3)) were used to find the relative potency of the drugs, the potency would be the same as in equation (2.7) if and only if the respective logistic curves are parallel (so that $\theta_{31}=\theta_{32}$ ); otherwise, the choice of the level $(50 \%, 75 \%$, etc. $)$ at which to compare the curves is paramount. As a result, we adopt as a sufficient condition in the assessment of relative potency the convention that the dose-response curves first be parallel. Regardless of whether or not the dose-response curves can be assumed to be parallel, use of the definition of relative potency given in equation (2.7) avoids any ambiguity.

Example 2.3.1 - By way of illustration, we examine the data given in Stokes et al (2000:331) to assess the relative potency of the two peptides. For this study, the authors assumed that the binomial-logistic model is appropriate with parallel curves and obtained the relative potency point estimate of $\rho=5.64$. Further, using an application of Fieller's Theorem (see Finney, 1971, p. 63), the authors also give an approximate $95 \%$ confidence interval for $\rho$ of $(1.53,20.07)$. Our analysis confirms that the likelihood-ratio test for parallelism dose retain the null hypothesis for these data, thereby supporting the inherent parallelism assumption. Also, this illustration highlights the fact that practitioners should not use the usual Wald intervals given by most software packages (e.g., the NLMIXED procedure in SAS ${ }^{\circledR}$ ) for providing an interval estimate of $\rho$ since this interval includes negative values here. The reliability of Wald intervals and indicators of their usefulness are discussed in O'Brien and Wang (1996) and Haines, O'Brien and Clarke (2004). Indeed, the much-preferred profile-based interval for the relative potency for these data is $(3.0,19.5)$, which is quite well approximated by the Fieller interval referenced above. We also point out that since the indicated scale for these data is the log-concentration (the Box-Cox parameter estimate is near zero), this example also highlights that were the researcher 
to incorrectly use the concentration scale, these data would then indicate non-parallelism of the dose-response curves, thereby rendering an examination of relative potency meaningless.

Once a reasonable model and scale are chosen and a reliable estimate of the relative potency of one drug to the other is obtained, the synergy experiment can be designed and the interaction can be assessed. Typical SAS ${ }^{\circledR}$ code is included in Appendix A.3.

\section{Assessing Synergy and Antagonism}

As noted above, our approach here is a parametric one in the sense that an underlying distribution, model and link functions are assumed known (and assessable via parametric lackof-fit tests); readers interested in a nonparametric approach can consult Kelly and Rice (1990). As noted in the previous section, the assessment of interaction between two or more drugs or similar compounds - as well as the optimal design strategies discussed in Section 5 - depends upon the model assumed for the specific application. Here, we discuss two very useful models and highlight the strengths and weaknesses of each.

\subsection{Assessment of Interaction using the Finney Model}

In some instances, the chosen design prohibits the use of a model as sophisticated as the separate ray model and necessitates the use of a model such as the Finney model discussed in section 2.1. Using this model, synergy is exhibited whenever the coefficient of synergy, $\theta_{5}$, is estimated as being significantly positive, antagonism is declared whenever it is significantly negative, and independent action is retained otherwise. This follows since if the synergy coefficient is negative, then $\mathrm{z}$ defined in equation (2.1) corresponds to the upper right portion of an ellipse; Appendix A.1 provides additional insight into these distinctions by relating the Finney and the separate ray model parameters. The following example provides a classic illustration of the use of the Finney model in agricultural research.

Example 3.1.1 - Gerig et al (1989) examined the interaction between the phenolic acids, ferulic $\left(\mathrm{x}_{1}\right)$ and vanilic $\left(\mathrm{x}_{2}\right)$ acid, on the growth of cucumber seedlings, in a study involving 3 chambers and only six support points per chamber, viz, $\left(\mathrm{x}_{1}, \mathrm{x}_{2}\right)=(0,0),(0,0.25),(0,0.50),(0.25,0),(0.50,0)$ and $(0.25,0.25)$ (in $\mu \mathrm{m} / \mathrm{g})$. Since only one interior point is included in the study design, use of a model more sophisticated than the Finney5 model given in equations (2.1) and (2.2) is not possible without additional, overly restrictive assumptions. Thus, we fit this model under the assumption of Gaussian, homoskedastic errors. For this model, the synergy coefficient, $\theta_{5}$, is estimated to be -0.8266 , leading one to suspect significant antagonism between these phenolic acids, and this indeed is the conclusion given in Gerig et al (1989). Although this conclusion may be justified, two caveats should be noted. First, that the chosen design has only one interior point, puts a great deal of weight on the underlying model assumptions in this instance; clearly, much more reliable results can be obtained with a more sophisticated design (see section 5 below). Second, although the corresponding Wald test of independence $\left(\theta_{5}=0\right)$ yields a p-value of $0.0009\left(t_{11}=4.517\right)$, the more reliable profile-based test of the same hypothesis results in a $p$ value of $0.0254\left(\mathrm{~F}_{1,11}=6.68\right)$. It follows that a more accurate conclusion here may be that these 
data show marginal or tentative evidence of antagonism and indicate that a larger, betterdesigned study is needed.

Although the previous example highlights the usefulness of the Finney statistical model in assessing the interaction of similar compounds in certain important settings, many other examples highlight the inadequacy of this model. For example, in several cases, use of this model resulted in large discrepancies between the actual and the predicted responses. Thus, in the next section, we introduce a richer statistical model - one which includes the Finney model as a special case or "sub-model."

\subsection{Assessment of Interaction using the Separate Ray Model}

One reason the Finney model fails to adequately fit certain interaction datasets is that for these datasets, the slopes are not the same along the various rays; see Appendix A.1 for algebraic connections between these models. A second, more important, reason for the inadequacy of the Finney model is that, whereas the Finney model assumes that a single coefficient of synergy is adequate over all combinations of two or more drugs, in many cases the degree of synergy or antagonism depends upon the relative proportions of the drugs in a mixture. This concept, which translates into the degree and nature of interaction depending for example on the slope $\left(\mathrm{c}_{\mathrm{r}}\right)$ in equation (2.4), viz, $x_{2}=c_{r} x_{1}$, is addressed in Tallarida (2000:9) and in Gessner and Cabana (1970). Using data examining the interaction between chloral hydrate $\left(\mathrm{x}_{2}\right)$ and ethanol $\left(\mathrm{x}_{1}\right)$, Gessner and Cabana (1970) presents evidence of significant synergy between these drugs for slopes greater than $\mathrm{c}=1 / 7$ and independent action for gentler slopes.

To make this concept of varying degrees of synergy for different rays more concrete, consider the so-called $\mathbf{L D}_{\mathbf{5 0}}$ line connecting $\theta_{21}$, the LD50 associated with drug A, with $\theta_{22}$, the LD50 associated with drug $B$. We express this line as

$$
\mathrm{x}_{1} / \theta_{21}+\mathrm{x}_{2} / \theta_{22}=1
$$

To illustrate, if the relative potency $(\rho)$ of substances A to B is equal to 10 (e.g.,, if $\theta_{21}=10$ and $\theta_{22}=1$, so that substance $\mathrm{B}$ is ten times as potent as substance $\mathrm{A}$ ), then the effective equipotent (EE) ray is one which includes 5 parts of substance $A$ and one-half part of substance $\mathrm{B}$, etc. It follows in general that this EE ray has slope $1 / \rho$. We therefore define the effective fraction, $f$, along the $\mathrm{r}^{\text {th }}$ ray $\left(\mathrm{x}_{2}=\mathrm{c}_{\mathrm{r}} \mathrm{x}_{1}\right)$ by

$$
\mathrm{x}_{1}=\mathrm{f} \theta_{21} \quad \text { and } \quad \mathrm{x}_{2}=(1-\mathrm{f}) \theta_{22}
$$

where $\mathrm{f}$ lies between 0 and 1 , and where $\mathrm{f}=1$ corresponds to ray 1 (horizontal axis $\mathrm{x}_{2}=0$ ) and where $\mathrm{f}=0$ corresponds to ray 2 (vertical axis $\mathrm{x}_{1}=0$ ); not surprisingly, $\mathrm{f}=1 / 2$ for the $E E$ ray.

Thus, rays are referenced here either by the corresponding slopes as in equation (2.4) or by the corresponding effective fraction as in (3.2), and one can switch from one to the other by using the relations 


$$
c=[1 / f-1] / \rho \text { and } f=1 /[1+c \rho]
$$

For example, since the relative potency for the study given in Gessner and Cabana (1970) is estimated to be 10.93 , the slope of $c=1 / 8$ referenced above corresponds to the effective fraction $\mathrm{f}=0.42$, meaning that this ray is not far from the effective equipotent ray $(\mathrm{f}=1 / 2)$. Indeed, although referencing rays in terms of their slopes may be useful in geometric and graphical terms, we note that it is more advantageous to develop experimental designs for synergy studies in terms of their corresponding effective fractions.

For a given ray (referenced by slope or by effective fraction), note that if the corresponding $\mathrm{LD}_{50}$ along this ray falls below (towards the origin) the $\mathrm{LD}_{50}$ line given in equation (3.1), then this gives evidence of synergy along this ray; analogously, the $\mathrm{LD}_{50}$ falling above the $\mathrm{LD}_{50}$ line indicates antagonism. In order to obtain a valid statistical test of significance, we reparameterize the separate ray model, shifting away from the $\theta_{2 \mathrm{r}}$ (the $\mathrm{LD}_{50}$ along the $\mathrm{r}^{\text {th }}$ ray) parameters and introduce a new model parameter that can be used to test for interaction. This is achieved by introducing the so-called combination index for the $\mathbf{r}^{\text {th }}$ ray, $\boldsymbol{\kappa}_{\mathbf{r}}$. Given that the $\mathrm{LD}_{50}$ along this ray is on a line parallel to the $\mathrm{LD}_{50}$ line in equation (3.1) but with the right hand side in (3.1) equal to $\kappa_{\mathrm{r}}$ instead of 1 , viz,

$$
\mathrm{x}_{1} / \theta_{21}+\mathrm{x}_{2} / \theta_{22}=\kappa_{\mathrm{r}}
$$

then we are interested in estimating the corresponding combination index $\left(\kappa_{\mathrm{r}}\right)$ for each ray and in testing whether each is significantly less than one (synergy), equal to one (independent action) or greater than one (antagonism). Finally, by combining the relation for the $\mathrm{LD}_{50}$ along the $\mathrm{r}^{\text {th }}$ ray

$$
\theta_{2 \mathrm{r}}=\mathrm{x}_{1}+\mathrm{x}_{2}=\left(1+\mathrm{c}_{\mathrm{r}}\right) \mathrm{x}_{1}
$$

with equations (2.4) and (3.4), we obtain the relation

$$
\kappa_{\mathrm{r}}=\left[\theta_{2 \mathrm{r}}\left(\theta_{22}+\mathrm{c}_{\mathrm{r}} \theta_{21}\right)\right] /\left[\theta_{21} \theta_{22}\left(1+\mathrm{c}_{\mathrm{r}}\right)\right]
$$

or equivalently,

$$
\theta_{2 \mathrm{r}}=\left[\theta_{21} \theta_{22}\left(1+\mathrm{c}_{\mathrm{r}}\right) \kappa_{\mathrm{r}}\right] /\left(\theta_{22}+\mathrm{c}_{\mathrm{r}} \theta_{21}\right)
$$

for each interior ray, $r=3,4, \ldots R$. A more informative manner of expressing the $r^{\text {th }}$ combination index may be by the equation

$$
\kappa_{\mathrm{r}}=\theta_{2 \mathrm{r}} /\left[\theta_{21} \mathrm{f}+\theta_{22}(1-\mathrm{f})\right]
$$

obtained from equations (3.1) - (3.7); this expression gives the ratio of the actual $\mathrm{LD}_{50}$ along the $\mathrm{r}^{\text {th }}$ ray to the point on the $\mathrm{LD}_{50}$ line where the ray hits this line. 
It follows that for a study involving $\mathrm{R}$ rays, fitting the separate ray model includes the estimation of $2 \mathrm{R}$ model parameters, viz, the R slopes $\left(\theta_{31}, \theta_{32}, \ldots, \theta_{3 \mathrm{R}}\right)$, the $\mathrm{LD}_{50}$ 's for each of the two drugs $\left(\theta_{21}\right.$ and $\left.\theta_{22}\right)$, and the $(R-2)$ combination indices $\left(\kappa_{3}, \kappa_{4}, \ldots, \kappa_{R}\right)$; the $L_{50}$ 's of the interior rays are simply functions of these latter parameters. Computationally, this can easily be achieved using for example the SAS ${ }^{\circledR}$ statistical package by either programming the likelihood in SAS/IML or by using the NLMIXED procedure. The above concepts are illustrated with the following examples.

Example 3.2.1 - Martin (1942) presents data regarding the interaction between two insecticides, deguelin $\left(\mathrm{x}_{1}\right)$ and rotenone $\left(\mathrm{x}_{2}\right)$, in isolation and in a 1:4 mixture (thus a single interior ray), when sprayed on Macrosiphoniella sanborni, the chrysanthemum aphis; these data are also examined in Finney (1971) and Greco and Lawrence (1988). The binomial logistic model is assumed here since the number of dead plants is recorded out of a fixed number (usually 50) for each dose combination. We find for these data that an appropriate scale is indeed the log-dose scale (i.e., that $\theta_{6}$ in equation (2.6) is near zero). Note that since the underlying design involves only a single interior ray, the Finney4 model is equivalent to the equi-slope separate ray model (see Appendix A.1). However, the full separate ray model is required here since the likelihood-based test of common slopes is rejected $\left(\chi_{2}{ }^{2}=10.93, p=0.0042\right)$, and, using this richer model, we note that these data exhibit significant synergy with an estimated combination index of $\kappa_{3}=0.7355$ $\left(\chi_{1}^{2}=14.3, p=0.0002\right.$ corresponding to the test of independence, $\left.H_{0}: \kappa_{3}=1\right)$. It is important to highlight the fact that since for these data the relative potency is estimated as $11.97 / 4.77=2.51$ and since $c_{3}=1 / 4$, the interior ray used here corresponds to the estimated effective fraction $\mathrm{f}=$ 0.62 . That is, we conclude that these data suggest significant synergism corresponding to this fraction and that extrapolation to other fractions is potentially precarious; should this is the goal of a study, additional fractions (corresponding to additional rays) should be added to the underlying study design.

Example 3.2.2 - Designs with one interior ray and involving binomial data are used in Collett (2003:113) (originally from Hewlett and Plackett (1950)) and in Finney (1952:149) (originally from LePelley and Sullivan (1936)). The Hewlett and Plackett study involved an insecticide trial in which flour beetles were sprayed with either DDT (dichloro-diphenyltrichloroethane) or $\gamma$ BHC ( $\gamma$-benzene hexachloride) or a 1:1 mixture of the two. For these data, a common Box-Cox parameter (c.f., equation (2.6)) is required, the common slope hypothesis $\left(\theta_{31}=\theta_{32}=\theta_{33}\right)$ is accepted, and synergy is indicated (estimated combination index of $\kappa_{3}=0.4555$ and test of $\mathrm{H}_{0}: \kappa_{3}$ $=1$ yields $\left.\chi_{1}{ }^{2}=208.8, p<0.0001\right)$. More specifically, significant synergy is observed along the ray with estimated effective fraction of $f=0.44$ (using equation (3.3)) since the relative potency is estimated as $4.90 / 3.83=1.28$. Evidence of significant synergy is less clear for the LePelley and Sullivan study, which involves two trials, each involving house-flies sprayed with the insecticides rotenone or pyrethrins alone or in a (respectively $1: 5$ or $1: 15$ ) mixture of the two. Analysis of these data requires distinct Box-Cox parameters for each of the rays, and significant synergy is observed for the first trial (likelihood test of $\mathrm{H}_{0}: \kappa_{3}=1$ yields $\chi_{1}{ }^{2}=54.1, \mathrm{p}<0.0001$ ), for which the estimated effective fraction is $\mathrm{f}=0.46$, but independent action is concluded for the 
second trial (likelihood test of $\mathrm{H}_{0}: \kappa_{3}=1$ yields $\chi_{1}{ }^{2}=0.8, \mathrm{p}=0.37$ ), for which the estimated effective fraction is $\mathrm{f}=0.70$. Thus, the LePelley and Sullivan studies highlights the fact that synergy can be observed for some mixture proportions but not for others.

Example 3.2.3 - In cases where the response variable is a percentage - for example, percent of weed control - a transformation such as the logit transform in equation (2.9) or the arcsinesquare root transformation is usually warranted. This is the case for the trials - each with a single interior ray - reported in Shelton (1990) and Tallarida (2000:63). Shelton (1990) examined percentage weed control after use of one of two herbicides or a 1:4 mixture of the two, and the separate ray model (with log-dose) with different slopes is found to be adequate using the logit transformation; these data exhibit significant synergy (estimated combination index of $\kappa_{3}=$ 0.4286 and test of $\mathrm{H}_{0}: \kappa_{3}=1$ yields $\chi_{1}{ }^{2}=30.6, \mathrm{p}<0.0001$ ) along the ray with estimated effective fraction of $\mathrm{f}=0.26$. That this estimated fraction is so low may be evidence that the relative potency was poorly estimated a priori (see equation (3.3)) and that perhaps a slope of $\mathrm{c}=1 / 10$ (a mixture of $1: 10$ ) should have been used. In contrast, the study reported in Tallarida (2000:63), which also gives evidence of significant synergy (with estimated combination index of 0.3232 which corresponds to the effective fraction of 0.51 ), includes an interior ray that is nearer to the corresponding effective equipotent rays $(f=1 / 2)$.

The previous example highlights one of the potential dangers of the use of only one interior ray in assessing the interaction of similar compounds - that if the relative potency is estimated very poorly, then the region of exploration actually studied could be quite different from the intended one. This follows since if $\rho_{0}$ is the initial a priori guess at the relative potency and $\mathrm{f}_{0}$ is the effective fraction corresponding to the chosen ray in equation (3.2), whereas $\rho_{\mathrm{F}}$ is the actual estimated relative potency for a given study, then the corresponding realized effective fraction, $\mathbf{f}_{\mathbf{F}}$, is given by

$$
\mathrm{f}_{\mathrm{F}}=\left[1+\mathrm{r}\left(1 / \mathrm{f}_{0}-1\right)\right]^{-1}
$$

where $r=\rho_{F} / \rho_{0}$ is the ratio of the final and guessed potencies. Thus, regardless of the value of the initial fraction $\left(f_{0}\right)$, the realized fraction $\left(f_{\mathrm{F}}\right)$ in (3.9) can take on any number between zero and one depending on the value of $r$. As a result, it is our strong recommendation that researchers first get an idea of the relative potency of the substances under study (stage I) before the actual (stage II) interaction study is performed; this two-stage design strategy is further discussed in section 5 below. Fortunately, one obvious way to (at least partially) circumvent the need for a precise estimate of the relative potency is to use a design with several interior rays, as was the case for the following illustrations.

Example 3.2.4 - Giltinan et al (1988) and Morgan (1992:17) discuss data gathered to study the interaction between two insecticides (labelled A and B) where the response variable is the number of dead tobacco budworms of a fixed total (usually 30) budworms exposed to either insecticide $A$ alone, insecticide $B$ alone or a combination in a mixture of $3: 1,1: 1$ or 1:3. For these data and the separate ray model, the indicated Box-Cox scale is the log-dose scale (c.f., 
equation (2.6)), the common-slope hypothesis $\left(\theta_{31}=\ldots=\theta_{35}\right)$ is accepted, and the estimated combination indices are $\kappa_{3}=1.57, \kappa_{4}=2.46$ and $\kappa_{5}=1.57$, thereby indicating significant antagonism between these insecticides. Since we can further accept that $\kappa_{3}=\kappa_{5}=\kappa$, the corresponding separate ray model contains only 5 parameters. It turns out too that the Finney 4 submodel (c.f., equation (2.3)) also adequately fits these data $\left(\chi_{1}{ }^{2}=3.8, p=5.13 \%\right)$, thereby implying that the estimated combination indices and $\mathrm{LD}_{50}$ 's follow the pattern as specified by the Finney model (see equation (7.2) in Appendix A.1); however, we feel that the separate ray model is more informative since it allows us to verify significant antagonism for each of the chosen rays. Finally, note that since the estimated relative potency here is $10.06 / 11.49=0.88$, the selected mixtures correspond to the realized effective fractions $f=0.28,0.53$, and 0.77 , and so the selected mixtures adequately cover the mixture region (i.e., f's between 0 and 1).

The previous example points out the far-reaching application and usefulness of the separate ray model in conjunction with several (typically three) interior rays; this methodology is easily extended to find estimated mixtures associated with the maximal amount of synergy or antagonism. We return to a discussion of the set-ups and experimental designs useful in interaction experiments in Section 5 below after a discussion of two noteworthy extensions of the basic separate ray model.

\section{Some Extensions of the Finney and Separate Ray Models}

The basic separate ray model discussed in Section 3 is easily adapted to account for blocking variables and for checkerboard designs, which are occasionally encountered in practical settings.

\subsection{Experiments Involving Blocks}

Agricultural studies are often laid out in growing chambers and herbicide trials typically involve distinct field plots; thus, it is important to note that variability associated with these blocking factors must be removed in the Finney or separate ray models so that potential interactions can more accurately be assessed. The following example illustrates how interaction patterns can better be discerned when this variability is first removed.

Example 4.1.1 - For the cucumber-phenolic acids dataset discussed above in Example 3.1.1, Gerig et al (1989) recommends that a separate upper-asymptote parameter $\left(\theta_{1}\right.$ in equation (2.2)) be used for each of the three chambers, and this results in the estimated coefficient of synergy given above $\left(\theta_{5}=-0.8266\right)$ and the tentative conclusion of significant antagonism (likelihood test of independence resulted in the p-value of $2.54 \%$ ). In effect, this procedure allows us to remove the fixed block effects as captured in the upper-asymptote parameter; an alternate strategy is to treat the chambers as random blocks and the realized upper asymptotes as random realizations from a random (e.g., Gaussian) distribution. This latter technique, which is easily implemented using the NLMIXED procedure in SAS ${ }^{\circledR}$, yields almost identical results in terms of the estimated synergy coefficient $\left(\theta_{5}=-0.8371\right)$, but for this random upper-asymptotes, the likelihood-based test of independence is strongly rejected $\left(\chi_{1}{ }^{2}=12.9, p=0.0003\right)$. It is therefore 
reasonable to conclude that these data do therefore suggest significant antagonism between these phenolic acids - though a better-designed study is clearly needed. We also underscore the importance of removing the random block (chamber in this example) variability so that interactions can be more clearly detected.

Similar results can be obtained in settings where randomised complete or incomplete block designs are used in environmental or agricultural field studies or in which individual subjects act as blocks (such as in medical studies).

\subsection{Horizontal and Vertical Rays; Checkboard Designs}

Although most recent interaction studies use the ray designs discussed in sections 2.1 and 3.2, occasionally the so-called checkerboard design (or some variant of it) is employed. Checkerboard designs combine rays $1\left(\mathrm{x}_{2}=0\right)$ and $2\left(\mathrm{x}_{1}=0\right)$ with a factorial design using all non-zero doses. For example, situations arise for which researchers are interested in determining whether significant interaction is present with herbicide $\mathrm{A}$ for fixed levels of herbicide $\mathrm{B}$ (called horizontal rays, of the form $\mathrm{x}_{2}=\mathrm{c}$ ) or with herbicide $\mathrm{B}$ for fixed levels of herbicide A (called vertical rays, of the form $\mathrm{x}_{1}=\mathrm{c}$ ). Thus, Gessner and Cabana (1970) describes a study in which synergy is detected for both horizontal and vertical rays. The following examples demonstrate two simple extensions of the separate rays model to handle these designs.

Example 4.2.1 - Whereas classic checkerboard designs include both the horizontal ray $\left(\mathrm{x}_{2}=0\right)$ and the vertical ray $\left(\mathrm{x}_{1}=0\right)$, the design used in Peters and Ganter (1935) (and discussed in Finney (1952:118)) involves only horizontal rays. These rays correspond to one of seven different concentrations of hydrocyanic acid in a study of the toxicity of hydrocyanic acid to the grain weevil Calandra granaria, in which batches of ten insects were exposed to the specific concentration for between two to five different exposure times. Thus, the authors are interested in testing for the interaction between concentration and exposure time, and the separate ray model is readily extended to handle this design, with details given in Appendix A.2. For this design and model, $\theta_{21}$ represents the $\mathrm{LD}_{50}$ for the lowest concentration $(3.5 \mathrm{~g} / \mathrm{cu} . \mathrm{m}$. $), \theta_{22}$ denotes the $\mathrm{LD}_{50}$ for the highest concentration $(30 \mathrm{~g} / \mathrm{cu} . \mathrm{m}$.), and combination indices again quantify the deviation of the data from the straight line connecting $\theta_{21}$ and $\theta_{22}$. Using this approach, these data show significant synergy for each of the concentrations $8.5,11.5,15.5$ and $24 \mathrm{~g} / \mathrm{cu}$.m with estimated combination indices, $\kappa=0.83,0.64,0.61$ and 0.76 , respectively. Since the vertical ray $\left(\mathrm{x}_{1}=0\right)$ is omitted here, the Finney model cannot be fit, and the extension of the separate ray model described here provides the only sensible means to detect interaction for these data.

\section{Experimental Design Strategies for Detecting Interaction}

Optimal designs are often chosen to maximize some function of the information matrix, $\mathbf{M}$, which corresponds to the assumed model function. Designs that maximize the determinant of $\mathbf{M}$, called D-optimal designs, are discussed here. The D-criterion is by far the most popular one (see Silvey (1980:40)), although other criteria are discussed in Atkinson and Donev (1992) and 
Atkinson and Haines (1996); a more non-technical introduction to optimal design strategies is provided in O'Brien (2003b) and O'Brien and Funk (2003).

Unfortunately, so-called optimal designs often only have enough support points to estimate the model parameters of the assumed model function, and hence provide no ability to test for lack of fit. Our approach here is to provide "robust" optimal designs - efficient designs with extra support points - by embedding key deviations into the model. Robust optimal designs are therefore advantageous since they (1) provide a nice compromise between the theoretical and the practical, (2) do not waste limited resources, (3) efficiently estimate model parameters, and (4) allow for lack of fit tests and tests of model discrimination.

As noted above in Example 3.2.3, we advise the use of a two-stage experimental design process - in the first stage a small study be set up to give an accurate idea of the relative potency (if one is not readily available from previous studies), and a second stage (using the relative potency estimate as an a priori estimate) to evaluate synergy. Thus, for example if the separate-ray binomial logistic model is to be used, then for the first stage, we intentionally saturate the model with extra parameters by allowing three-parameter logistic models on each of the first and second rays. This process of saturation provides designs with additional design points to check for model mis-specification. An alternate strategy is to find optimal designs using a set of initial parameter estimates at the ends of a reasonable range of such values. Our design strategy includes the choice of a stage I design that uses the compromise subset D-criterion (see O'Brien (2003b)) and which focuses on maximizing the information regarding the relative potency parameter ( $\rho$ in equation (2.7)). Once the relative potency has been efficiently estimated, phase II of our design strategy suggests using ray designs with the single-compounds rays $\left(\mathrm{x}_{1}=0\right.$ and $\mathrm{x}_{2}=0$ ) with 3-5 interior rays if possible, and with additional support points chosen either by using a saturated model such as the power logistic model or by using two sets of initial parameter estimates. To illustrate, for the phenolic acid illustration discussed in Example 3.1.1, this strategy would require a phase I design with at least seven support points and a phase II design with at least ten support points, and so may very well mean the use of incomplete blocks if limitations exist on the number of runs per chamber.

\section{Summary and Further Extensions}

This paper highlights statistical modelling and experimental design strategies useful in characterizing the interaction between two or more similar compounds with a focus on agricultural applications. These model parameters and designs are easily obtained using software packages such as the SAS ${ }^{\circledR}$ system, and NLMIXED and SAS/IML programs are available in Appendix A.3 or from the author upon request. Further extensions of these methods - with applications to two- and three-drug therapies - are currently underway. 


\section{Appendices}

\section{A.1. The Equivalence of the Finney 4 and the Equi-slope Separate Ray Models}

As noted in section 2.1, the Finney4 model defined in equations $(2.1)-(2.3)$ is identical to the separate ray model defined in sections 2.1 and 3.2 when the respective slope parameters, $\theta_{31}, \theta_{32}$, $\ldots, \theta_{3 \mathrm{R}}$, are equal (and equal to $\theta_{3}$ from the Finney4 model). This result is established by further relating $\theta_{2}, \theta_{4}$, and $\theta_{5}$ (Finney model) and $\theta_{21}, \theta_{22}, \ldots, \theta_{2 R}, \kappa_{3}, \ldots, \kappa_{R}$ (separate ray model) according to the relationships $\theta_{2}=\theta_{21}, \theta_{4}=\theta_{21} / \theta_{22}=\rho$ from equation (2.7), and

$$
\theta_{5}=\left(1-\kappa_{\mathrm{r}}\right) \phi_{\mathrm{r}}\left(\theta_{21} \theta_{22}\right)^{1 / 2}
$$

for $r=3,4, \ldots, R$ and where $\phi_{r}=\left(1+c_{r}\right) /\left[\theta_{2 r} c_{r}^{1 / 2}\right]$. Since the term $\phi_{r}\left(\theta_{21} \theta_{22}\right)^{1 / 2}$ in equation (7.1) is positive, this equation highlights the fact that synergy is indeed achieved in the Finney4 model whenever $\theta_{5}>0$ (since this occurs when $\kappa_{\mathrm{r}}<1$ ), that independent action occurs whenever $\theta_{5}=0\left(\kappa_{\mathrm{r}}=1\right)$, and that antagonism occurs whenever $\theta_{5}<0\left(\kappa_{\mathrm{r}}>1\right)$. Equation (7.1) also highlights that in addition to the equi-slope assumption, the Finney4 model also includes the restriction amongst the $\kappa_{\mathrm{r}}{ }^{\mathrm{s}} \mathrm{s}$,

$$
\left(1-\kappa_{\mathrm{s}}\right) \phi_{\mathrm{s}}=\left(1-\kappa_{\mathrm{t}}\right) \phi_{\mathrm{t}}
$$

for all (unequal) $\mathrm{s}$ and $\mathrm{t}$ chosen from $\{3,4, \ldots, \mathrm{R}\}$ whenever the data is chosen using at least two interior rays.

\section{A.2. The Extended Separate Ray Model for Horizontal and Vertical Rays}

With reference to a design analogous to that used in Example 4.2.1 and using the separate ray model with rays $1\left(x_{2}=0\right)$ and $2\left(x_{1}=0\right)$ with respective $L D_{50}$ 's $\theta_{21}$ and $\theta_{22}$, for the horizontal ray $x_{2}=b_{1}$ with $L D_{50}, \theta_{2 b 1}$, we have $\theta_{2 b 1}=x_{1}+x_{2}$, so that $x_{2}=\theta_{2 b 1}-b_{1}$. Defining the interior combination index $\kappa_{\mathrm{b} 1}$ as in equation (3.4), we obtain horizontal combination index

$$
\kappa_{\mathrm{b} 1}=\theta_{2 \mathrm{~b} 1} / \theta_{21}+\mathrm{b}_{1}\left(\theta_{21}-\theta_{22}\right) / \theta_{21} \theta_{22}
$$

A similar calculation for the vertical ray $x_{1}=a_{1}$ and $\theta_{2 a 1}$ the $L D_{50}$ on this ray gives the following form for the vertical combination index

$$
\kappa_{\mathrm{a} 1}=\theta_{2 \mathrm{a} 1} / \theta_{22}-\mathrm{a}_{1}\left(\theta_{21}-\theta_{22}\right) / \theta_{21} \theta_{22}
$$

Extending this for a horizontal ray design as used in Example 4.2.1, with $\theta_{21}$ and $\theta_{22}$ representing the $\mathrm{LD}_{50}$ 's for the lowest value of $\mathrm{x}_{2}\left(\mathrm{x}_{2}=\mathrm{c}_{1}\right)$ and the highest value of $\mathrm{x}_{2}\left(\mathrm{x}_{2}=\mathrm{c}_{2}\right)$ respectively, the line connecting these two points has slope $\left(c_{1}-c_{2}\right) /\left(\theta_{21}-\theta_{22}\right)$. Thus, for the (interior) horizontal ray $x_{2}=c_{r}$ with $L_{50} \theta_{2 r}$, the horizontal combination index is defined here by 


$$
\kappa_{\mathrm{r}}=\theta_{2 \mathrm{r}} / \alpha+\mathrm{c}_{\mathrm{r}} / \beta
$$

with $\alpha=\left(c_{2} \theta_{21}-c_{1} \theta_{22}\right) /\left(c_{2}-c_{1}\right)$ and $\beta=\left(c_{2} \theta_{21}-c_{1} \theta_{22}\right) /\left(\theta_{21}-\theta_{22}\right)$.

\section{A.3. Typical SAS ${ }^{\circledR}$ Code for Assessing Relative Potency and Synergy}

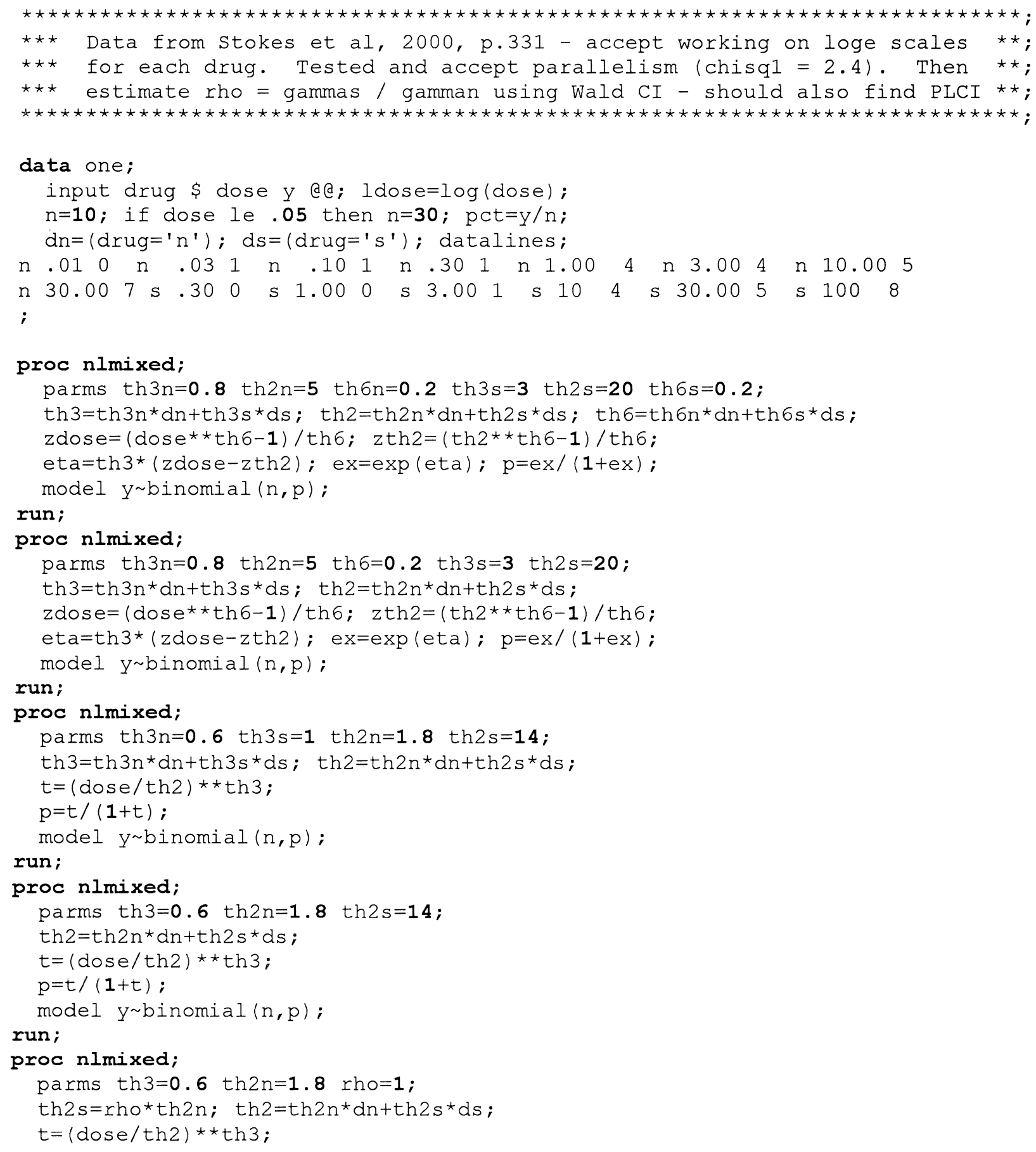




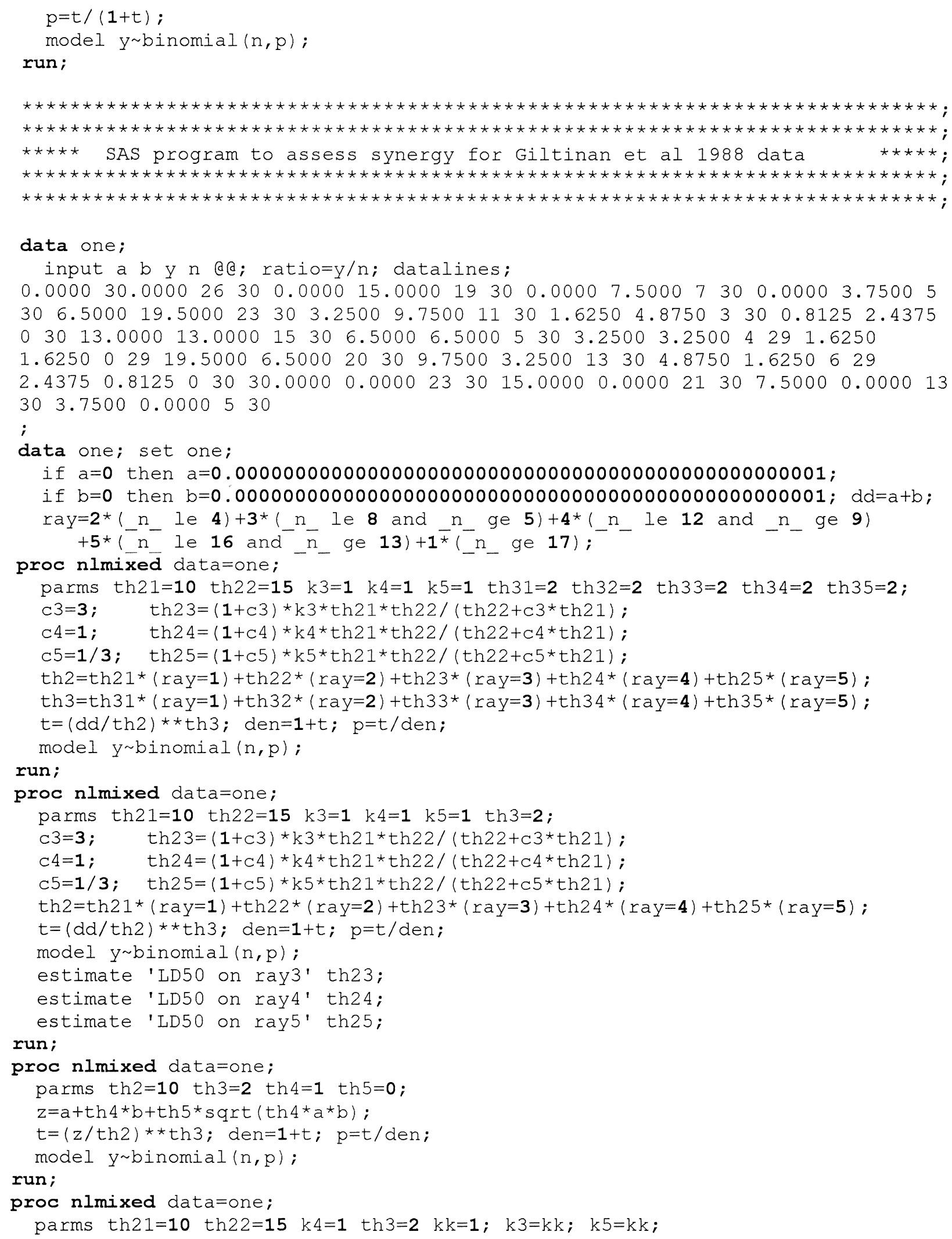




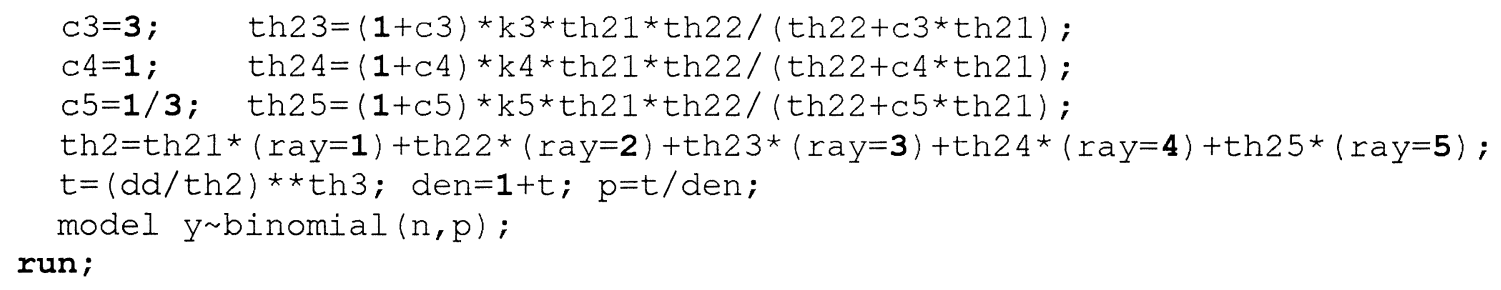

\section{Acknowledgements}

The author gratefully acknowledges the technical assistance of Luc Bijnens, Jacky van Dun, and Luc Wouters, as well as the financial support of Janssen Pharmaceutica N.V. (Beerse, Belgium) and Limburgs Universitair Centrum (Diepenbeek, Belgium).

\section{References}

Abdelbasit, K.M. and Plackett, R.L., 1982, Experimental Design for Joint Action, Biometrics, 38, 171-179.

Atkinson, A.C. and Donev, A.N., 1992, Optimum Experimental Designs (Oxford Statistical Series, No. 8), Clarendon Press, Oxford.

Atkinson, A.C. and Haines, L.M., 1996, Designs for Nonlinear and Generalized Linear Models, in Ghosh, S. and Rao, C.R., eds., Handbook of Statistics, Vol. 13, Elsevier Science, Leiden, 437475 .

Barton, C.N., Braunberg, R.C. and Friedman, L., 1993, Nonlinear Statistical Models for the Joint Action of Toxins, Biometrics, 49, 95-105.

Bates, D.M. and Watts, D.G., 1988, Nonlinear Regression Analysis and its Applications, Wiley, New York.

Berenbaum, M.C., 1989, What is Synergy?, Pharmacological Reviews, 41, 93-141.

Chou, T-C. and Talalay, P., 1981, Generalized Equations for the Analysis of Inhibitions of Michaelis-Menten and Higher Order Kinetic Systems with Two or More Mutually Exclusive and Nonexclusive Inhibitors, Eur. J. Biochem., 115, 207-215.

Collett, D., 2003, Modelling Binary Data, $2^{\text {nd }}$ Edition, Chapman \& Hall, London.

Cox, C., 1992, A GLM Approach to Quantal Response Models for Mixtures, Biometrics, 48, 911-928. 
Dawson, K.S., Carter Jr., W.H. and Gennings, C., 2000, A Statistical Test for Detecting and Characterizing Departures From Additivity in Drug/Chemical Combinations, J. Agric., Biol., \& Environ. Statistics, 5, 3, 342-359.

Finney, D.J., 1952, Probit Analysis, $2^{\text {nd }}$ edition, Cambridge University Press.

Finney, D.J., 1971, Probit Analysis, $3^{\text {rd }}$ edition, Cambridge University Press.

Finney, D.J., 1978, Statistical Methods in Biological Assay, $3^{\text {rd }}$ edition, Charles Griffin \& Company, London.

Gerig, T.M., Blum, U. and Meier, K., 1989, Statistical Analysis of the Joint Inhibitory Action of Similar Compounds, J. Chemical Ecology, 15, 10, 2403-2412.

Gessner, P.K. and Cabana, B.E., 1970, A Study of the Hypnotic and of the Toxic Effects of Chloral Hydrate and Ethanol, J. Pharmacol. Exp. Ther., 174, 247-259.

Giltinan, D.M., Capizzi, T.P. and Malani, H., 1988, Diagnostic Tests for Similar Action of Two Compounds, Applied Statistics, 37, 1, 39-50.

Govindarajulu, Z., 2001, Statistical Techniques in Bioassay, $2^{\text {nd }}$ edition, Karger, Basel.

Greco, W.R. and Lawrence, D.D., 1988, Assessment of the Degree of Drug Interaction Where the Response Variable is Discrete, ASA JSM Biopharmaceutical Section Proceedings, 226-231.

Greco, W.R. and Tung, Y., 1991, D-Optimal Experimental Designs for Quantifying Synergy, ASA JSM Biopharmaceutical Section Proceedings, 244-249.

Greco, W.R., Bravo, G. and Parsons, J.C., 1995, The Search for Synergy: A Critical Review from a Response Surface Perspective, Pharmacological Reviews, 47, 2, 331-385.

Haines, L.M., O’Brien, T.E. and Clarke, G.P.Y., 2004, Kurtosis and Curvature Measures for Nonlinear Regression Models. Forthcoming in Statistica Sinica, Vol. 14.

Hewlett, P.S. and Plackett, R.L, 1950, Statistical Aspects of the Independent Joint Action of Poisons, Particularly Insecticides. II. Examination of Data for Agreement with the Hypothesis, Annals Applied Biology, 37, 527-552.

Hutchinson, C.M., McGiffen Jr., M.E., Ohr, H.D., Sims, J.J. and Becker, J.O., 2000, Efficacy of Methyl Iodide and Synergy with Chloropicrin for Control of Fungi, Pest Management Science, $56,413-418$.

Kelly, C. and Rice, J., 1990, Monotone Smoothing with Application to Dose-Response Curves and the Assessment of Synergism, Biometrics, 46, 1071-1085. 
Khinkis, L.A. and Greco, W.R., 1993, Analytical Solution of the D-Optimal Design Problem for Studies of Drug Synergy, ASA JSM Biopharmaceutical Section Proceedings, 323-327.

LePelley, R.H. and Sullivan, W.N., 1936, Toxicity of Rotenone and Pyrethrins, Alone and in Combination, J. Econ. Ent., 29, 791-796.

Martin, J.T., 1942, The Problem of the Evaluation of Rotenone-Containing Plants. VI. The Toxicity of 1-Elliptone and of Poisons Applied Jointly, with Further Observations of the Rotenone Equivalent Method of Assessing the Toxicity of Derris Root, Annals Applied Biology, 29, 69-81.

McCullagh, P. and Nelder, J.A., 1989, Generalized Linear Models, $2^{\text {nd }}$ Edition, Chapman \& Hall, London.

Morgan, B.J.T., 1992, Analysis of Quantal Response Data, Chapman \& Hall, London.

Myers, R.H., Montgomery, D.C. and Vining, G.G., 2001, Generalized Linear Models: With Applications in Engineering and the Science, Wiley, New York.

O’Brien, T.E., 2003a, Class Notes for 'Statistical Modelling and Design for Bioassay and Drug Synergy', Limburgs Universitair Centrum (Diepenbeek, Belgium).

O'Brien, T.E., 2003b, Practical Design Strategies for the Detection of Drug Synergy, Electronic Notes in Discrete Mathematics, 15, available online at: http://www.elsevier.nl/gej-ng/31/29/24/ 75/23/78/endm15056.pdf

O’Brien, T.E. and Funk, G.M., 2003, A Gentle Introduction to Optimal Design for Regression Model, The American Statistician, 57, 4, 265-267.

O'Brien, T. E. and Wang, Y., 1996, Using SAS Software to Assess and Adjust for Nonlinearity in Nonlinear Regression Models, SUGI 21 Proceedings, Cary, NC: SAS Institute, 1274-1283.

Peters, G. and Ganter, W, 1935, Zur Frage der Abtoetung des Kornkaefers mit Blausaeure, Z. angew. Ent., 21, 547-558.

SAS Software, Version 8.2, 2001. Software and online help at www.sas.com.

Seber, G.A.F. and Wild, C.J., 1989, Nonlinear Regression, Wiley, New York. Kansas State University 


\section{Applied Statistics in Agriculture}

Stokes, M.E., Davis, C.S. and Koch, G.G., 2000, Categorical Data Analysis Using the SAS System, Second Edition, SAS Institute, Cary, N.C.

Tallarida, R.J., 2000, Drug Synergism and Dose-Effect Data Analysis, Chapman \& Hall/CRC, Boca Raton, FL. 